\title{
A Dialogue about the Nature and Unification of Information Science and Information Philosophy ${ }^{\dagger}$
}

\author{
Kun Wu ${ }^{1}$, Pedro Marijuan ${ }^{2}$ and Zhensong Wang ${ }^{1, *}$ \\ 1 School of Humanities and Social Sciences, Xi'an Jiaotong University, Xining West Road, No. 28, \\ Xi'an 710049, China; wukun@mail.xjtu.edu.cn \\ 2 Bioinformation Group, Aragonese Institute of Health Sciences, Center for Biomedical Research of Aragon \\ (CIBA) Avda. San Juan Bosco, 13, Floor 0, Zaragoza 50009, Spain; pcmarijuan.iacs@aragon.es \\ * Correspondence: zhensong1167@gmail.com; Tel.: +86-183-9261-9539 \\ + Presented at the IS4SI 2017 Summit DIGITALISATION FOR A SUSTAINABLE SOCIETY, Gothenburg, \\ Sweden, 12-16 June 2017.
}

Published: 8 June 2017

\begin{abstract}
At the invitation of Kun $\mathrm{Wu}$, Head of the International Center for the Philosophy of Information at the $\mathrm{Xi}^{\prime} \mathrm{An}$ Jiaotong University, Pedro C. Marijuan paid a ten-day academic visit to the University 4-14 November 2016. During his visit, Marijuan presented three lectures to students and teachers and had two dialogues with Wu November 11th and 12th on the topic of "The Nature and Unification of Information Science and Information Philosophy". Under this main topic, several sub-topics were discussed, such as the origin and development of information science and technology, the concept of entity, the fundamental principles for building a unified information science, relevant bio-information studies, the epistemological media of information philosophy, the relationship between information flows and matter-energy flows, the statuses of computationalism and general information theory, the structure of unified information science and so on. This extended abstract succinctly displays some basic content of those dialogues.
\end{abstract}

Keywords: information science; information philosophy; nature; unification

\section{Introduction}

This dialogue aims at presenting essential questions and relevant possible solutions about unified information science. Finally, discussants came to a consensus in the following points: (1) The understanding of the concept of information should be at the level of meta-science and meta-philosophy; (2) The premise for the unification of information sciences is a unified information philosophy; (3) Computationalism cannot take the lead in the process of building a unified information science; (4) A unified information science must be built through criticizing and transcending the current situation of the information sciences; (5) The communication among diverse disciplines and fields within the scope of the information sciences can and should be improved by reference to information philosophy; (6) For its research, a unified information science needs a unified object-information entity (the "informosome", previously defined by Professor Wu); (7) A unified information science also requires a unified mode of information thinking. This dialogue has a great significance on the study of unified information science.

W: Nice to meet you, Prof. Pedro Marijuan. Could you please present some comments firstly on the nature and unification of information science and information philosophy, which we are collectively interested in and concern about, so that we can have further discussion.

P: Thank you very much for your gracious invitation. Very generally I divided the modern development of science-technology into three main phases, and each of those three phases had its own representative sciences and technological objects. The representatives in the first phase are 
classical mechanics and both the clock and the telescope, and in this phase, the realism of entities and the experimental discovery of laws of nature is the great achievement of science. The representatives in the second phase are thermodynamics and the steam engine, and it is in this phase when we find the concept of energy and energy-flow, and we also find that the relation between energy and entity is just like the relation between fish and water: everything, everywhere. The main representatives in the third phase are information science and the computer. And in this third phase, the synthetic development will integrate different theories and disciplines scattered in many information fields into a unified information science.

W: I think the first two historical phases that you described should belong to a same phase, and you miss a phase. I suppose that the phase of electric energy and nuclear energy is the second phase of development of human science and technology. In fact, in the level of development of science and technology, the corresponding three phases embodied three scientific paradigms and three scientific thinking modes. And they respectively are: entity realism and entity thinking mode, field and energy realism and energy thinking mode, information system complexity synthesis theory and information thinking mode. Otherwise, what is the scientific foundation of information technology origin? If we stay at the level of computer science, it is impossible to expound the scientific principles behind that information technology. Actually, since the middle of nineteenth century, the second law of thermodynamics has introduced the ideas of structure, relation and evolution into physics, which are the exact contents that information thinking mode concerns.

P: Thinking about the principles of information phenomena, we will face at least these basic entities: subject, object, and their mutual interrelationship of communication patterns-plus the customary observer. Concerning human individuals, they have a general openness to the environment and they are particularly open to communicate with each other. Both the information flow and the energy flow are related to the process of self-creation and self-maintenance of the informational entities, which makes the organization of their "life cycles" or life courses possible.

W: Can we describe information by using the concept of entity? From perspective of philosophy and history of science, the concept of entity was primarily used on describing matter, and the following developed energy thought and information thought led to some kind of non-entity explanation theories.

P: Your opinion is reasonable. It is just in my personal knowledge system, and it has a different meaning with the traditional philosophical concept of entity. Here, what I call entity has to be understood as an informational organization. I just adopt "entity" to symbolize the pure subject, object, and interrelationship of informational phenomena a(nd particularly the subject).

$\mathrm{W}$ : I suggest using the "informosome" (or information system) instead of the concept of information entity, which is conducive to avoid some conceptual confusion. The information has a deeper meaning waiting to be explained, which is more comprehensive and incisive than its explanation in bio-information science or other scientific fields. We need to define it in the level of meta-science and meta-philosophy. And the definition on this level is to make sure the information has a relatively independent status in the ordinary ontology, and reveal the more general meaning and value of information.

P: Yes, there are many schools in the world that can be related to that deeper meaning, to what I call the foundations of information science. What we need now is advancing toward a unified new thinking mode, which will help us to avoid confusion brought by hundreds of definitions from different disciplines and fields.

W: When you talk about the difficulty of synthesis in the concrete scientific levels, it is understandable. But, if we rise to an abstract level, is the synthesis still difficult? The synthesis does not mean that every detail should be included, which is impossible. The synthesis is a process of abstraction and generality extraction.

P: I agree with you, the philosophy should be the upmost abstraction field. And also we need a new way of thinking which may be provided by the philosophy. There are two approaches for the development of information science at present: On the one side, there is an approach we may call 
weak approach. In this approach, the computationalism would lead information science. On the other side, there would be the strong approach. It stresses the necessity of a unified way of thinking to reveal the operation mechanisms of information flows in different realms.

W: The computer is only a tool adopted by artificial intelligence, which cannot symbolize all fields and disciplines in information science domain, and also cannot symbolize the existing mode and operation mode of information in the universe and our world. According to the origin of information science, the information science does not originate from computer technology. As an existence that is distinguished with material phenomena, the information's ontological status, meaning and value far exceed those of concept of number and break the narrow fence of computationalism.

P: You are quite right. I believe that information means different things to different people or scientists with diverse knowledge backgrounds. Otherwise, I believe that the epistemology is hard to be unified. And what we need is a meta-epistemology, an epistemology that can complete the unification.

W: The information medium theory of epistemology in our information philosophy system just is this kind of meta-epistemology. And it proposes that the cognition is constituted by five kinds of mediums: first, the medium of information field emitted by objects; second, the medium of human physiological structure; third, the medium of human cognitive structure, which is the different academic backgrounds that you just mentioned, like biology and physics; Fourth, the medium of material instruments; fifth, the medium of natural history and social history, which include the origin of life and the environment of the following individual development. And each one of those five mediums is the selection, transformation, matching and reconstitution of information. And the information in these mediums will also permeate into the process of human cognition, when they are involved into human cognition.

P: Those five mediums theory is fresh to me. I think any of those mediums would have unlimited information, I mean they are open-ended. For any individual, it is impossible to grasp even one tenth of their specific information, which means that we will face some serious difficulties on this kind of study. The main difficulty is that the content waiting to be studied is extremely excessive and heterogeneous. I suggest we have another angle to continue our investigation, which is the information flow that I mentioned. It has a unique nature, notwithstanding its possible multiplicity of appearances. For me, I prefer to study the information flow from the perspective of science.

W: The five mediums that I mentioned belong to the content of science. If we generally treat them as information flow, we would not interpret the specific process of cognition. In the other words, we would not know how many times the information structure in the cognition has been changed, transformed and reconstituted. On the scientific study on cognition, we had better not neglect all the mediums by using a single general concept-information flow.

P: But the information flow could be easily testified by science. And it is useful for our specific scientific study on information activities in different scientific fields and disciplines.

$\mathrm{W}$ : That is true. We do not deny the existence of information flow. And we can say that every detail of those five mediums has the trace of information flows. Our discussion speaks volumes. And we make much consensus on many aspects. And of course, we also have differences in some viewpoints.

P: Ok. We have a lot of deep discussion today. And some of your viewpoints are innovative and make me exciting that a philosophy of information and a science of information can meaningfully "inform" each other. I am so delighted!

Conflicts of Interest: The authors declare no conflicts of interest. 